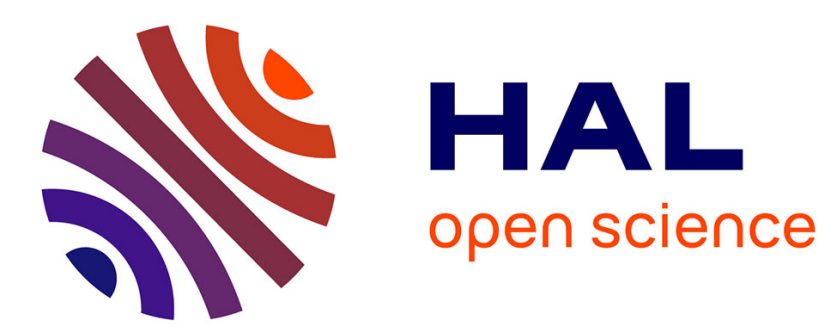

\title{
Application of PAMS Collaboration Platform to Simulation-Based Researches in Soil Science: The Case of the MIcro-ORganism Project
}

Khanh Nguyen Trong, Benoit Gaudou, Tuong Vinh Ho, Nicolas Marilleau

\section{To cite this version:}

Khanh Nguyen Trong, Benoit Gaudou, Tuong Vinh Ho, Nicolas Marilleau. Application of PAMS Collaboration Platform to Simulation-Based Researches in Soil Science: The Case of the MIcroORganism Project. Computing and Communication Technologies, 2009. RIVF '09. International Conference on, 2009, pp.1-8. 10.1109/RIVF.2009.5174623 . hal-00741738

\author{
HAL Id: hal-00741738 \\ https://hal.science/hal-00741738
}

Submitted on 17 Oct 2012

HAL is a multi-disciplinary open access archive for the deposit and dissemination of scientific research documents, whether they are published or not. The documents may come from teaching and research institutions in France or abroad, or from public or private research centers.
L'archive ouverte pluridisciplinaire HAL, est destinée au dépôt et à la diffusion de documents scientifiques de niveau recherche, publiés ou non, émanant des établissements d'enseignement et de recherche français ou étrangers, des laboratoires publics ou privés. 


\title{
Application of PAMS collaboration platform to simulation-based researches in Soil science: The case of the MIcro-ORganism project
}

\author{
Trong Khanh Nguyen*, Benoit Gaudou*, Tuong Vinh $\mathrm{Ho}^{*}$ and Nicolas Marilleau ${ }^{\dagger}$ \\ * UMI 209 UMMISCO, Institut de la Francophonie pour l'Informatique (IFI), Ha Noi, Viet Nam \\ Emails: ntkhanh@ifi.edu.vn, benoit.gaudou@alumni.enseeiht.fr, ho.tuong.vinh@auf.org \\ $\dagger$ UMI 209 UMMISCO, Institut de Recherche pour le dveloppment (IRD), Bondy, France \\ Email: nicolas.marilleau@ird.fr
}

\begin{abstract}
Major researches in the domain of complex systems are interdisciplinary, collaborative and geographically distributed. The purpose of this paper is to explore a new collaborative approach that facilitates scientists' interactions during the modelling and simulation process. Based on the problem of collaboration in a simulation project, we identify the needs for this kind of platform. Accordingly, a new collaborative platform dedicated to agent-based simulation (PAMS) has been developed. This new environment integrates common collaborative tools (e.g. videoconferencing, instant messaging, whiteboard) and specific tools to share and manipulate models, simulators, experiments and results... In this paper, we illustrate our approach by presenting our experiment with MIOR, a model developed in soil science.
\end{abstract}

Index Terms-collaborative simulation, agent-based simulation, distributed systems.

\section{INTRODUCTION}

Modelling and simulation of complex systems are by nature interdisciplinary and collaborative activities. Data collection, model conceptualization and their implementation using computational tools require close teamwork amongst various players such as domain experts, modelers and computer scientists. Indeed it is very unusual that such specialists are geographically located at least in the same city. Internet and modern communication media allow these kinds of distant collaborations, but specific tools are nevertheless required to improve their efficiency.

The advent of new information technologies and communications tools has enabled the development of a plethora of collaborative platforms [1]. A few of them, such as BSCW [2], E-Groupware [3] and Sakai [4], have positioned themselves as collaboration-oriented extensions of generalist communications platforms in the scientific world. These products integrate specific functionalities facilitating: (i) access to knowledge and scientific information, (ii) interaction and collaboration between researchers, and (iii) a more effective dissemination of research results. Such platforms are still relatively undeveloped. Most of these collaboration systems are limited to data repositories with web interfaces [5] and thus remain incapable of supporting significant collaborative efforts despite growing demands for such tools in the scientific community [6].
This paper focuses on the modelling and simulation domain. We illustrate scientists' needs for collaborative tools by taking the MIOR model [7] as example. MIOR is an agent-based model developed to simulate the decomposition process of organic residues by micro organisms in the soil. But the demand is similar in most simulation projects such as gamavi [8] (epidemiology), Ichthyop [9] (halieutic), Miro [10] (geography) and Sworm [11] (soil science).

As most of modelling and simulation projects, MIOR has required a deep cooperation between researchers from different disciplines such as ecology and computer science. As usual scientists were geographically separated: for example the initial MIOR model war elaborated in cooperation between researchers from 2 continents, 3 countries and 4 cities. In this hard working environment, the need of efficient collaborative tools appears obvious.

The aim of this paper is to present our attempt to give some answers to the collaboration problem. The main idea is to place models, simulators, experiments and results at the center of the collaboration. From this idea, we have designed and developed methodologies and a web-based "groupware", named "PAMS", containing common collaborative tools (videoconferencing, instant messaging, and so on) and specific tools dedicated to the simulation domain (sharing experiments, results, experience exchange...) aiming at supporting collaboration between domain experts, modelers and computer scientists.

The paper is structured as follows. Section II presents the MIOR model. In Section III we analyze the collaboration problem and describe some existing tools aiming at answering this issue. Section IV presents our own answer attempt and details the architecture of PAMS. Finally in Section $V$ we compare our approach with other existing ones and show how our application can address collaboration problems involved in the MIOR use.

\section{THE MIOR MODEL}

The soil is an extremely complex and heterogeneous environment composed of organic and mineral aggregates between which can live bacteria colonies. The aim of the MIOR project [7] is to model and simulate the process of mineralization 
in the soil, i.e. the decomposition by micro organisms of substrates organic matter. This model gives the opportunity to study phenomena such as the decomposition of litter in the natural environment or the $\mathrm{CO}_{2}$ efflux produced during this process by the microbial respiration [12], actual experiments of this process being indeed really hard to carry out.

The main idea underlying MIOR model is to focus, among various identified factors, on the influence of the population of microorganisms and in particular on their biochemical composition [13] or on their spacial repartition and spatiotemporal dynamics $[14]^{1}$. The MIOR model is original among existing ones because it uses an agent-based approach to model this process. In particular, this paradigm allows it to represent explicitly the heterogeneity of the soil and the spatio-temporal dynamics of living and non-living organic matter, contrarily to mathematical models such as CANTIS [16], SOMKO [17] or Fontaine et al. 's one [18].

From the MIOR model, a simulator has been developed ex nihilo using Net Logo framework. This model can carry out simulations and investigate the influence of various parameters on the biological process. It has been improved during more than four years and is become quite elaborated. But in return it is quite hard to take in hand for a new user that does not well know this programming language. This point limits the possible diffusion of this model and its use by other researchers.

\section{THE COLLABORATION IN THE SIMULATION OF COMPLEX SYSTEMS}

\section{A. The collaboration problem in a collaborative simulations project: the case of MIOR}

1) The collaboration problem in the case of the MIOR model: Results of this model can be useful for biologists coming from various communities of soil sciences. For example, this model is used to study:

- microbial dynamics [7]

- macrofaune (earthworms) impact on organic matter distribution [11], [19]

- mychorizial phenomena.

This model could be used in other case studies and should be known by other the soil science communities. But, the MIOR model is a standalone NetLogo model that needs computer science qualification to be used. In addition, it is difficult to give an accurate presentation of a model in an paper, and thus it is impossible for readers to reproduce the model. Only simulation results can be published and discussed into an article. Thus, researches around models such as MIOR cannot be diffused with efficiency throughout soil sciences communities.

2) A first attempt with Web-Sim-MIOR: To promote the MIOR model and facilitate exchanges around it, a participative portal, called WEB-SIM-MIOR ${ }^{2}$, has been created and

\footnotetext{
${ }^{1}$ Note that [15] showed that the surface of contact between living and nonliving organic matters has a great impact on the decomposition process.

${ }^{2}$ The WEB-SIM-MIOR project has been initiated by Christophe Cambier.
}

published at http://www.ird.fr/websimmior. This web site was a first attempt to provide collaborative web tools dedicated to model and simulate complex systems.

The WEB-SIM-MIOR portal is based on the generic NetLogo platform and allows users to run simulators on their own computer via their web browser without installing anything. They can also download simulators if they want to launch simulations offline using hardware resources of their own computer. Moreover registered users of a WEB-SIM-MIOR portal have the opportunity to upload a NetLogo simulator and to publish it with a short and long descriptions.

Up today, the MIOR model is the only published one on the WEB-SIM-MIOR portal. Around this model a tutorial promote agent-based modelling in soil sciences. During the project, sessions and training have been given to biologists and experts in soil sciences. The experience of these meetings shows us:

- thematicians' needs to use modelling and simulation technics to study complex systems;

- the lack of knowledge of modelling and simulation approaches;

- the biologists' need to exchange around their researches.

Obviously, the experience of the WEB-SIM-MIOR project shows several weakness of the existing web portal such as:

- the impossibility to setup simulators that are not based on NetLogo,

- the poverty of proposed collaborative tools;

- the needs to download and run simulators on the user's computer (simulator complexity are constrained by users' computer limitations).

It follows that the WEB-SIM-MIOR portal is not the ideal response to the problems of collaboration and scientific sharing in the domain of soil sciences or in other research areas.

3) The collaboration problem in the general case: A simulation project is a teamwork rather than the task of a single and isolated scientist [20]. In this context, a collaborative platform takes an important role in the success of such a project: "collaboration-enabling tools are critical for transforming simulations into true research modalities." [19]. But, among existing groupwares only few are dedicated or useful to support collaboration in modelling and simulation.

From the experience of WEB-SIM-MIOR and other works about collaborative systems, it is possible to imagine what is the ideal groupware to simulate complex systems. An ideal simulation groupware must be:

- compliant with major simulation platforms to facilitate the setup of simulators coming from famous simulation platforms (NetLogo, Repast, MadKit, ... );

- generic to permit simulation of various complex systems;

- user friendly to be used by scientists coming from various research areas;

- a distributed system to support large scale simulations and many simultaneous connections and simulations;

- synchronous to permit instant exchanges between scientists (videoconferencing, instant messaging, white board, desktop sharing, ... ); 


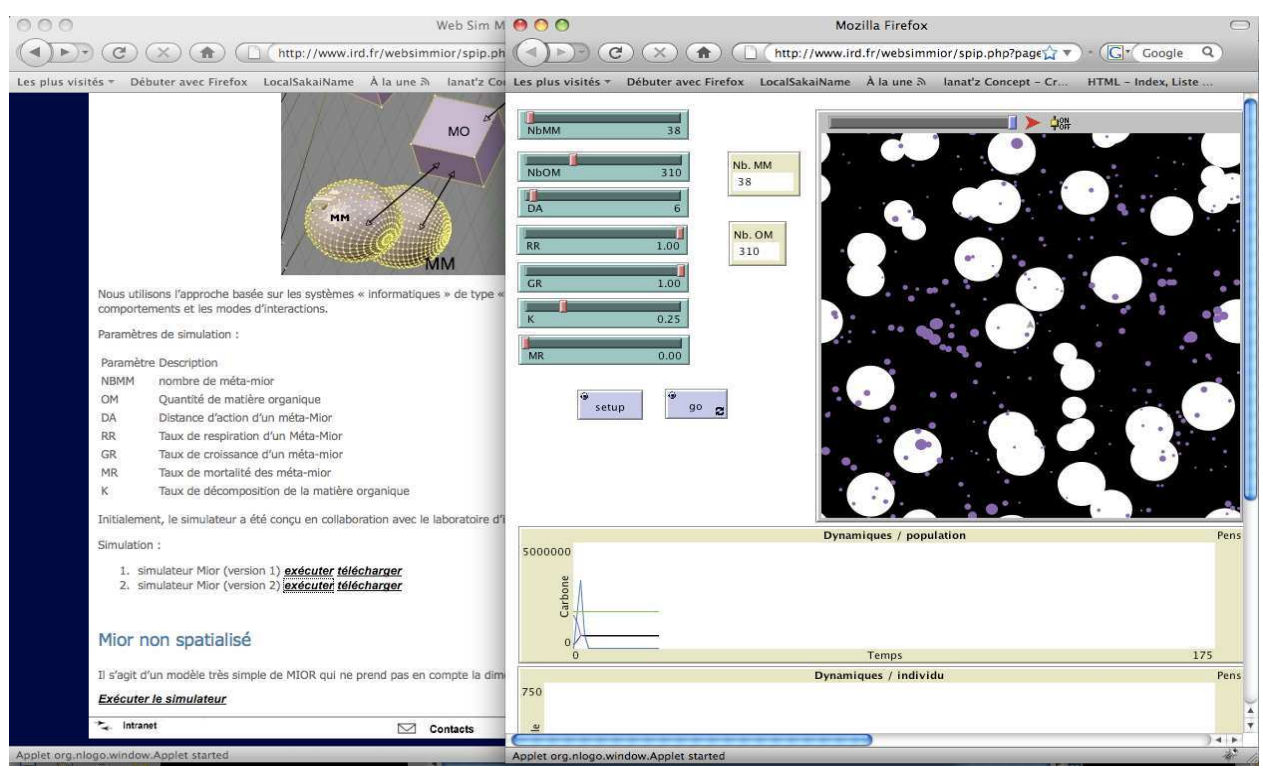

Fig. 1. WEB-SIM-MIOR

- asynchronous to allow different time collaboration (mail, forum, wiki, ...);

- based on web technologies to be available everywhere without installing anything;

- composed of basic simulator functionalities to start, stop and setup simulations with various parameters;

- composed of advanced functionalities to analyze simulation results, store results in a database and replay simulations;

- composed of collaboration features dedicated to the simulation domain (parameters setting, result sharing, ...);

- composed of public-private information management system to allow a participant to determine a public part (shared with each other) and a private part (kept for himself) of his own simulation desktop.

Note that the previous enumeration is not an exhaustive list of desiderata necessary for having an ideal simulation groupware.

\section{B. Existing tools}

The advent of new information technologies and communication during the past fifteen years has enabled the development of a plethora of collaborative platforms named BSCW (Basic Support Cooperative Work). Major BSCWs are dedicated to (i) industrial use [21], [4], [5], (ii) the field of tele-medicine [1] or (iii) the e-learning. These platforms allow people to collaborate via the Web in order to (i) create and manage documents and to (ii) exchange information and ideas during meetings. For this purpose, these groupwares support various tools such as videoconferencing, instant messaging, resources sharing, white board and so on.

At the intersection of web technologies, collaborative systems and simulation domain, a new way had emerged during the last decade [20]. It promotes new solutions named Web-
Based Simulation Groupware (WBSG) which incorporate simulators into a collaborative environment.

Major WBSGs are dedicated to the education domain. As example we present ERCIS [22] and DomoSim-TPC [23] frameworks.

ERCIS is "a prototype Group Distance Exercise system that [...] provide[s] addfordable distributed interactive simulation" [22]. This WBSG is applied to military simulations. It permits to train in the use of RBS-70 missile units [22] in a virtual environment which takes into account (i) geographical information, (ii) aircraft characteristics and (iii) exercise scenario. ERCIS allows to create sessions including 11 participants. They collaborate via a browser and applets embedded. But, ERCIS does not support several basic collaborative tools such as instant messaging, forum and so on.

DomoSIM-TPC environment is used for collaborative learning of domotical design. This environment allows collaboration during the modelling and the simulation processes. To create their model, students work together through shared workspaces. To simulate it, they use a Shared Window System, such as NetMeeting@. Contrary to ERICS, DomoSIM-TPC includes several collaboration tools such as: (i) mail, (ii) generic and structured instant messaging, (iii) decision-making tool, (iv) tele-pointers, (v) tele-data, and so on. But it does not support videoconferencing, desktop sharing and many asynchronous collaborative functions such as: forum, wiki, blog...

Only few WBSGs are dedicated to research works, for example to the study of complex systems. In this domain, we can cite Web-Sim-MIOR presented in the previous section. But this tool is more an interactive web portal than a groupware. It allows to run only NetLogo simulators. Users can only interact with the browser and they cannot communicate and run simulations together. 
The Web Based Simulation Center (WBSC) framework [5] provides "a concept and a prototypical solution for supporting specialists from different fields who are involved in a simulation project, phase by phase, supported by Web-based resources" [5]. It supports all steps of a collaborative simulation project. One advantage of this platform is to provide sufficient functionalities for collaboration such as an instant messaging, a file management system, an environment for collaborative writing of documents (Collaw [5]), and special features to simultaneously realize experiments (e.g. to modify parameters, execute a simulation and display results as images...).

But WBSC only supports the simulator GPSS/H [24]. It cannot integrate new simulators that have been written in other simulation systems such as the MIOR model. Moreover WBSC does not support synchronous collaborative tools such as videoconferencing, whiteboard or screen sharing...

In [20], Korichi Ahmed et al. present a BSCW usable in various research domains. It associates a BSCW system with external softwares that allow to realize synchronous collaborative functions. For this purpose, this groupware proposes a shared workspace with asynchronous collaborative features (e.g. forums, version management, event service) and synchronous tools using an external software close to MS NetMeeting. The latter introduces services such as videoconferencing, instant messaging, desktop sharing, software sharing, and so on.

The approach proposed by Korichi Ahmed et al. is very simple, useful and generic. But, among its drawbacks, we can note that no privacy rules can be defined. Every participant of a session can control the simulation. In addition there is no generic feature (e.g. a database) allowing to store simulation results: results are shared by the group during the simulation and cannot be reused afterwards.

\section{Needs}

As far as we are aware, there does not exist any framework that supports functionalities required by a generic and efficient WBSG. Major groupwares are not dedicated to the simulation domain and do not support the use of simulators and simulation platforms. Existing WBSCs are not ideal solutions because they do not support every requirement identified in Section III-A3.

From the lack of existing WSBCs and desiderata presented in Section III-A3, it appears that there is a need to develop a generic WBSC giving the opportunity (i) to execute various kinds of simulators on a server, (ii) to share simulator control, (iii) to share simulation results and (iv) to manipulate simulation results during and after the running of the simulator.

We propose in the sequel a new WBSC based on the idea that simulators can be viewed as a collaborative object manipulated via a didactic and user-friendly web interface.

\section{PRESENTATION OF THE PAMS FRAMEWORK}

\section{A. Principle: PAMS a new collaborative framework for agent- based simulation of complex system}

PAMS project introduces a new approach to collaborate in a modelling and simulation project. With PAMS, models and simulators are considered as objects shared by a group of researchers, which is possible to manipulate, configure, analyze and so on over a web interface. PAMS is indeed an environment enabling researchers to work together on the design of models and / or on their exploration (execution of models based on various scenarios, i.e. interactions conceptualized in the context for which the model has been designed or execution of a specific simulation).

To reduce the developing time and cost, we focus on open source solutions which provide some generic collaborative tools and which are possibly extensible with new modules needed for collaboration in the field of complex systems simulations. After an investigation, we have chosen Agora [25] which provides synchronous tools (such as videoconferencing, whiteboard, chat...) and Sakai [25] which supports asynchronous tools (such as file management, forum...). And we have added to it new collaborative modules dedicated to simulation activities, such as: (i) setting simulations, (ii) executing simulations on a remote server, (iii) visualizing and analyzing results, (iv) managing versions of available models, (v) archiving experiments and results, (vi) annotating experiments and results (giving contextual comment), (vii) storing image or graph outputs of the simulation.

PAMS uses various generic, independent and popular platforms to manage multi-agent simulations. In particular, our application supports Repast [26], Gama [8] and NetLogo [27]. The web interface allows users to upload their own models for these simulators and to laugh some collaborative simulations. Note that PAMS is not dedicated to some particular simulators and from the developers point of view, it is quite easy to integrate to our application some new simulators thanks to its modular architecture presented in the next section.

\section{B. Architecture: PAMS - a modular environment}

PAMS framework is a distributed system based on common components (see Fig.2):

- A web application server based on Jsp, Ajax and Servlet (Tomcat \& Sakai) to manage the collaborative web interface that displays models, experiments, simulators and results with a simple, adaptable and didactic structure of data.

- A distributed component based application server (EJB-Jonas) to manage the groupware kernel (execute simulators, manage experiments, inputs, outputs and collaboration).

- A Database (MySQL) to store users, experiments and results.

Fives modules compose the kernel of the PAMS environment: 


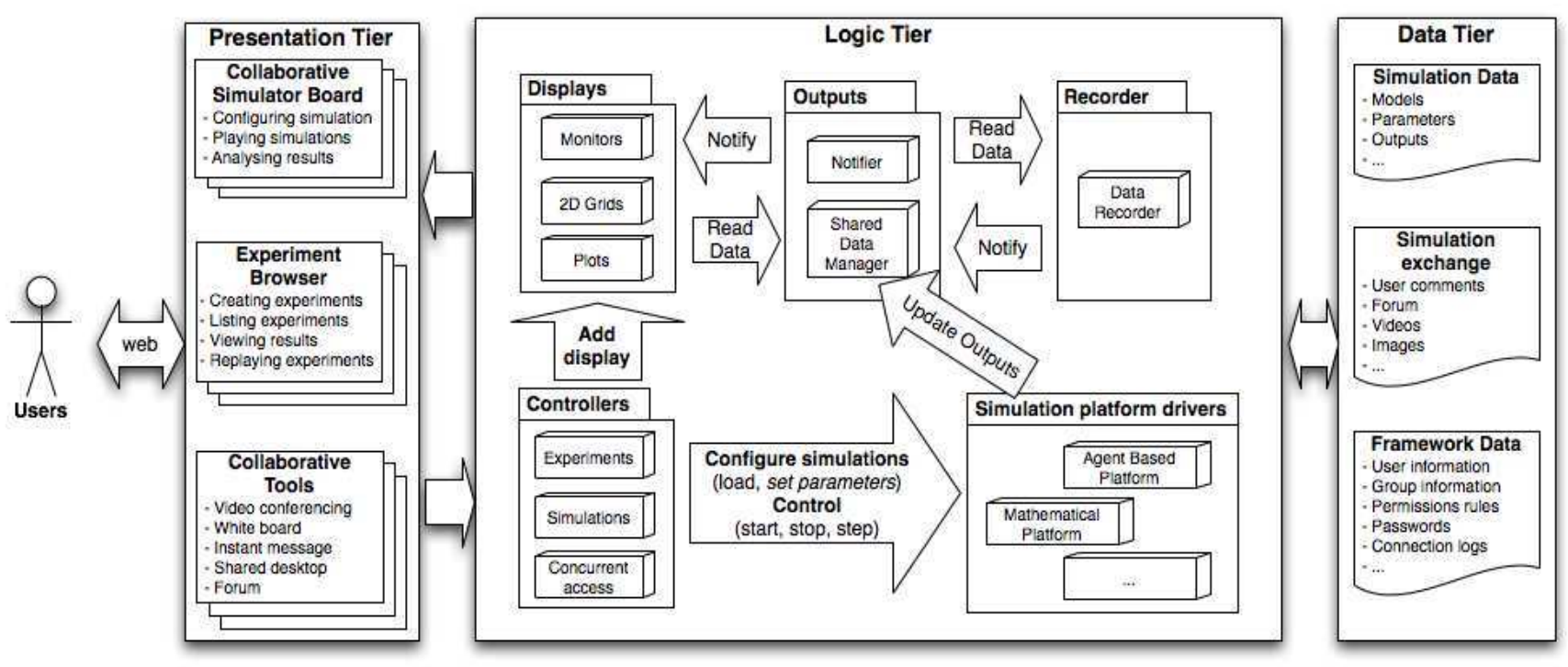

Fig. 2. PAMS logical architecture

- Simulation platform drivers package contains the kernel of agent-based platforms as Repast [26], Gama [8] and NetLogo [27].

- Outputs package manages simulation results coming from simulation platforms, and shares these data with other modules of the systems.

- Displays package formats shared outputs in order to generate and manage user displays: monitors, plots and/or 2Dgrids (images).

- Recorder package saves in a database every value that an output has taken during a simulation. These data are read by the experiment browser web interface.

- Controllers package aims at managing experiments and simulators and at ensuring the coherency and concurrency of objects shared by users (parameters, simulation outputs, experiments).

Controller and Display packages are the only modules available outside the kernel of PAMS framework. Through these two modules, graphical user interfaces (GUI) allow, with a collaborative scheme, to load and manipulate (start, stop, parameter settings, and so on) simulators, and to customize simulation outputs by adding/removing monitors, plots, grids and so one.

PAMS environment proposes a generic web based collaborative GUI. This interface takes advantage of typical collaborative tools (videoconferencing, white-board and so on) coming from Sakai and Agora tools. In addition, PAMS provides functions dedicated to the simulation domain:

- A collaborative simulation board for executing and sharing remote simulators

- An experiment browser for managing and replaying realized experiments and exchanging results.

Up to now, PAMS supports simulators coming from three agents based platforms: Repast, Gama, and NetLogo. Some famous and simple simulators have been deployed, e.g. the life game, Enn for Repast, Life for Gama and MIOR for NetLogo, to test PAMS functionalities. But others can easily be uploaded on the server via the web interface.

\section{CASE STUdy}

\section{A. Example of PAMS use with MIOR model}

The aim of this case study is to show how a group of scientists can use PAMS environment to work collaboratively on MIOR simulations. We consider as example the case of Mr. X and Mr. Y, researchers in ecology, respectively located in Paris and Hanoi. They wish to execute some simulations in order to get results to illustrate an article they are writing together.

They begin by connecting to a MIOR simulator via the PAMS platform, that is supposed to be running on a server. After the identification step, they access to their private workspace. Mr. X wants to create a simulation. From a list of available simulators, he selects the MIOR one. A new display appears showing information about it: aims of the model, inputs, outputs and so one. On this screen, Mr. X can see all public experiments done with the MIOR simulator. But, he prefers to create a new one. For that, $\mathrm{X}$ inputs a comment about the new experiment (its aim) and selects participants from a list of subscribed persons. In the case of this scenario, Mr. X selects Mr. Y. and submits the form. The experiment is now created. $\mathrm{X}$ is waiting Y's connection to start simulations.

Y selects also the MIOR simulator from the list of available simulators. Information about this simulator are displayed, and $\mathrm{Y}$ sees that $\mathrm{X}$ has invited him to participate in an experiment. $\mathrm{Y}$ connects to the experiment.

$\mathrm{X}$ and $\mathrm{Y}$ are seeing the same display: the simulation board of the MIOR simulator. $\mathrm{X}$ wants to start entering simulation parameters. He thus takes the token allowing the control of 


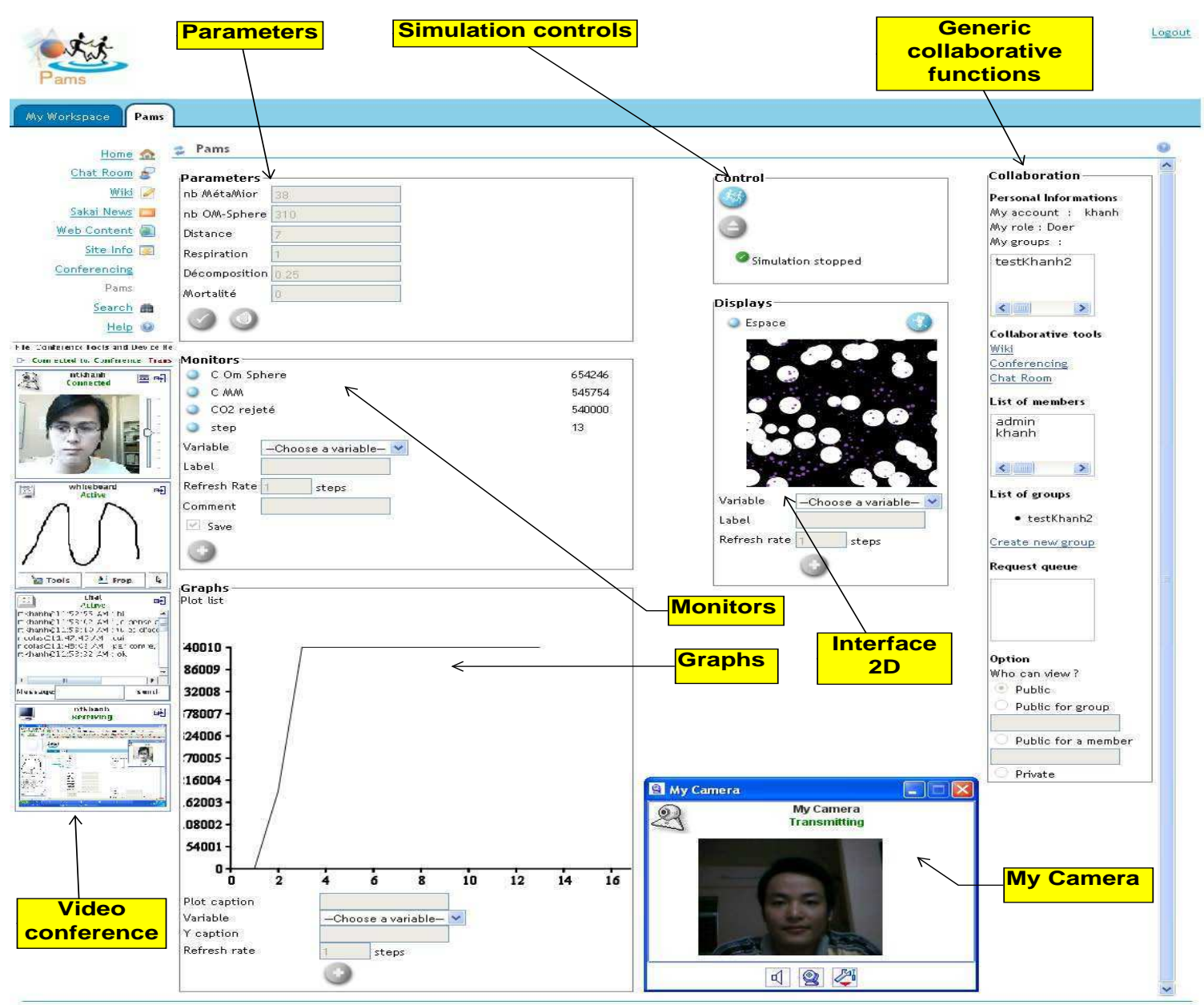

Fig. 3. MIOR under PAMS

the simulation. Y's display is frozen. He cannot perform any action on the simulator board, but sees modifications. After doing modifications, $\mathrm{X}$ releases the token. $\mathrm{Y}$ does not agree with X's parameters modifications. To explain his disagreement, Y starts a videoconferencing session integrated in the Agora meeting tool. Thanks to video, audio and whiteboard tools, $\mathrm{Y}$ discusses with $\mathrm{X}$. To support his remarks, $\mathrm{Y}$ shows an article by sharing his desktop and convinces $\mathrm{X}$. $\mathrm{X}$ thus takes again the token and changes parameters values according to Y's recommendation. Then, X starts the simulation.

$\mathrm{X}$ and $\mathrm{Y}$ can observe real-time changes in predetermined outputs. During the simulation, they discuss the evolution of the outputs. From these results, $\mathrm{X}$ and $\mathrm{Y}$ begin an analysis and make hypothesis about the phenomena they see. Due to time constraints Mr. X must leave. They both decide to resume this discussion later. So they record the session. Three days later, returning to the site, the conversation starts and resumes in the state where it had been left earlier...

\section{B. Comparison with existing tools}

This section will lead a comparison between five simulation platforms in which collaborative work is allowed: WEB-SIM-
MIOR, BSCW [5], ERICS [22], DomoSim-TPC [23] and PAMS. This study is summarized in Table I.

With regard to the modelling task, we can identify four classes of systems. In the first one, platforms support tools for modelling, but only one user can participate at a moment, it is a case of individual modelling. In the second class, shared modelling, platforms also support for modelling, but in addition all participants have a same view of the tools and can simultaneously model. model editing tool means that platforms allow to modify models. With model installer feature, platforms can be extended with others model from various simulators (e.g. Repast [26] models, NetLogo [27] models... ).

With respect to the simulation phase, we can distinguish seven features. For the mode of simulation launch, we identify the batch mode and the graphical mode. About the way to simulate, platforms can either allow individual simulation, in which only one user can simulate, distributed simulation, when each participant launches a simulator that is part of a global simulation [23], or shared simulation when all participants have the same view of the model and can simultaneously simulate. We also distinguish systems that allows to manipulate 
parameters and results (modify and set the parameters, view and analyze the results...) and to manage simulations (join a simulation, replay a simulation... ).

For generic collaborative functions, we study asynchronous functions such as: discussion forum, wiki, mail, bog... and following synchronous collaborative functions:

- Videoconferencing: Does system support videoconferencing?

- Chat tool

- Whiteboard

- Desktop sharing

In addition, we add two cases determining whether the platform is system independent and if it requires the installation of additional packages to use it.

In relation to the modelling task, there are two groups: a group allowing the modelling that includes DomoSim-TPC and BSCW, while the other group composed by PAMS, WEBSIM-MIOR and ERCIS does not support it. With PAMS and DomoSim-TPC users can add new models.

Concerning the simulation phase, every platform supports graphical mode while only BSCW allows to run simulation in batch mode. Individual simulation can be carried out by every platform, but shared simulation only by PAMS, BSCW and DomoSim-TPC and distributed simulation by ERCIS. Users can manipulate simulation with all platforms, however, only PAMS and DomoSim-TPC allow to manage simulation.

PAMS and BSCW strongly support for synchronous and asynchronous functions, but BSCW depends on system operation, and its use needs to install additional packages which can restraint its use. In general, for collaborative modelling and simulation DomoSim-TPC, BSCW and PAMS are best. But for research works, PAMS is more convenient, because DomoSimTPC is used to study, while BSCW is system dependent.

\section{CONCLUSION}

We have argued in this paper that a modelling and simulation work is by nature a collaborative task: it needs the participation of various domain experts that are usually geographically separated. The need of specific tools appears thus obvious. We have illustrated this point by taking the example of the MIOR project. We have highlighted that existing tools allowing collaborative work, in particular in the field of modelling and simulation, are still far from what we consider as being desiderata for an ideal WBSC. We thus have proposed a new collaborative framework dedicated to modelling and simulation called PAMS.

PAMS framework is based on an original approach in which the simulator is the support of the collaboration. It is a shared object manipulated by every member of a scientific group. We consider that users only need to view a simulator as a "black box". Members can thus concentrate and collaborate on its inputs and outputs. To help their collaboration, the GUI contains common asynchronous and synchronous tools such as videoconferencing, instant-messaging, whiteboard, and so one. Moreover PAMS provides to scientists a large number of collaborative tools dedicated to simulation. The web-based GUI allows them to access collaboratively to remote simulators and to shared experiments. This GUI also supplies collaborative functions to setup simulators, execute simulators on a remote server, visualize and analyze simulation results, and keep logs of each experiment.

On a simple but actual example using the MIOR model, we have shown that the use of PAMS: on the one hand, (i) addresses the problem posed by the geographically dispersed location of researchers and on the other hand, (ii) brings a new dimension to the simulation activities of complex systems.

PAMS is still under development. Up today it supports three agent-based simulation platforms (Repast, Gama and NetLogo). The modularity of PAMS permits our environment to be improved in many ways. Adding a new simulation platform only needs the creation of a new driver which is a quite low-cost task. New simulation platforms or standalone simulators will thus be integrated in the future. Modularity also allows a quite simple integration of new kinds of displays or collaborative tools. PAMS framework will thus be improved in many ways: (i) by adding new collaborative tools (e.g. an annotating system to comment experiments), (ii) by optimizing the system (e.g. adding load-balancing strategies), and (iii) by supporting new agent based simulation platforms (e.g. Madkit and so on). We also plan to test the existing version on a concrete research project applied to, for instance, geography or epidemiology. Feedbacks from these tests will provide the vital keys required to further developments and improvements of the PAMS environment.

\section{ACKNOWLEDGMENT}

This work was funded by the French IRD SPIRALES research program.

\section{REFERENCES}

[1] D. S. Voirin, "Contribution à la modélisation et à l'analyse des systèmes coopératif : application à la e-maintenance," Ph.D. dissertation, Université de Franche-Comté, 2006.

[2] T. Horstmann and R. Bentley, "Distributed authoring on the web with the bscw shared workspace system," StandardView, vol. 5, no. 1, pp. 9-16, 1997.

[3] R. Becker, B. Becker, M. Knotte, and I. KreiBlemeyer, Manual eGroupware 1.4., 1st ed., Creative Commons, 2007.

[4] C. Severance, J. Hardin, G. Golden, R. Crouchley, A. Fish, T. Finholt, B. Kirschner, J. Eng, and R. Allan, "Using the sakai collaborative toolkit in e-research applications. 19:12, 1643-1652 (2007)," Concurrency and Computation: Practice and Experience, vol. 19, no. 12, pp. 1643-1652, 2007.

[5] J. Henriksen, P. Lorenz, A. Hanisch, S. Osterburg, and T. Schriber, "Web based simulation center: professional support for simulation projects," in Winter Simulation Conference (WSC'02), vol. 1, 2002, pp. 807 - 815.

[6] K. Ahmed and B. Brahim, "Towards a web based simulation groupware: Experiment with bscw," Information Technology Journal, vol. 1812, no. 5638, pp. 332-337, 2008

[7] D. Masse, C. Cambier, A. Brauman, S. Sall, K. Assigbetse, and J.-L. Chotte, "MIOR: an individual-based model for simulating the spatial patterns of soil organic matter microbial decomposition," European Journal of Soil Science, vol. 58, no. 9, pp. 1127-1135, October 2007.

[8] E. Amouroux, C. Quang, A. Boucher, and A. Drogoul, "GAMA: an environment for implementing and running spatially explicit multi-agent simulations," in 10th Pacific Rim International Workshop on MultiAgents (PRIMA), Thailand, 2007. 
TABLE I

COMPARISONS BETWEEN PLATFORMS

\begin{tabular}{|c|c|c|c|c|c|c|}
\hline & & $\begin{array}{c}\text { WEB- } \\
\text { SIM-MIOR }\end{array}$ & BSCW & PAMS & ERCIS & $\begin{array}{l}\text { DomoSim- } \\
\text { TPC }\end{array}$ \\
\hline \multirow{4}{*}{ Modelling } & Individual modelling & & $\mathrm{X}$ & & & $\mathrm{X}$ \\
\hline & Shared modelling & & $\mathrm{X}$ & & & $\mathrm{X}$ \\
\hline & Model editing tool & & $\mathrm{X}$ & & & $\mathrm{X}$ \\
\hline & Model installer & & & $\mathrm{X}$ & & $\mathrm{X}$ \\
\hline \multirow{7}{*}{ Simulation } & Batch Mode & & $\mathrm{X}$ & & & \\
\hline & Graphical mode & $\mathrm{X}$ & $\mathrm{X}$ & $\mathrm{X}$ & $\mathrm{X}$ & $\mathrm{X}$ \\
\hline & Individual simulation & $\mathrm{X}$ & $\mathrm{X}$ & $\mathrm{X}$ & $\mathrm{X}$ & $\mathrm{X}$ \\
\hline & Distributed simulation & & & & $\mathrm{X}$ & \\
\hline & Shared simulation & & $\mathrm{X}$ & $\mathrm{X}$ & & $\mathrm{X}$ \\
\hline & $\begin{array}{c}\text { Parameters and results } \\
\text { manipulation }\end{array}$ & $\mathrm{X}$ & $\mathrm{X}$ & $\mathrm{X}$ & $\mathrm{X}$ & $\mathrm{X}$ \\
\hline & Simulation management & & & $\mathrm{X}$ & & $\mathrm{X}$ \\
\hline \multirow{7}{*}{$\begin{array}{l}\text { Generic } \\
\text { functions }\end{array}$} & Videoconferencing & & $\bar{X}$ & $\mathrm{X}$ & & \\
\hline & Chat tool & & $\mathrm{X}$ & $\mathrm{X}$ & & $\mathrm{X}$ \\
\hline & Whiteboard & & $\bar{X}$ & $\mathrm{X}$ & & $\mathrm{X}$ \\
\hline & Desktop sharing & & $\mathrm{X}$ & $\mathrm{X}$ & & \\
\hline & Asynchronous Functions & & $\mathrm{X}$ & $\mathrm{X}$ & & \\
\hline & System Independent & $\mathrm{X}$ & & $\mathrm{X}$ & $\mathrm{X}$ & $\mathrm{X}$ \\
\hline & $\begin{array}{c}\text { Need of Additional } \\
\text { packages }\end{array}$ & & $\bar{X}$ & & & \\
\hline
\end{tabular}

[9] C. Lett, P. Verley, C. Mullon, C. Parada, T. Brochier, P. Penven, and B. Blanke, "A lagrangian tool for modelling ichthyoplankton dynamics," Environmental Modelling \& Software, vol. 23, no. 9, pp. 1210-1214, 2008.

[10] A. Banos, S. Chardonnel, C. Lang, N. Marilleau, and T. Thevenin, "Simulating the swarming city: a mas approach," in procs. of The 9th Int. Conf. on Computers in Urban Planning and Urban Management, CUPUM 2005, London, UK, 2005.

[11] E. Blanchart, N. Marilleau, A. Drogoul, E. Perrier, J. Chotte, and C. Cambier, "SWORM: an agent-based model to simulate the effect of earthworms on soil structure," EJSS. European Journal of Soil Science, vol. 60, pp. 13-21, 2009.

[12] C. C. Cleveland, D. R. Nemergut, S. K. Schmidt, and A. R. Townsend, "Increases in soil respiration following labile carbon additions linked to rapid shifts in soil microbial community composition," Biogeochemistry, vol. 82, no. 3, pp. 229-240, 2007.

[13] C. Cambier, M. Bousso, D. Masse, and E. Perrier, "A new, offer versus demand, modelling approach to assess the impact of microorganisms spatio-temporal population dynamics on soil organic matter decomposition rates," Ecological Modeling, vol. 209, no. 2-4, pp. 301313, 2007.

[14] P. Garnier, C. Cambier, M. Bousso, D. Masse, C. Chenu, and S. Recous, "Modeling the influence of soil-plant residue contact on carbon mineralization: Comparison of a compartmental approach and a $3 \mathrm{~d}$ spatial approach," Soil Biology \& Biochemistry, vol. 40, no. 11, pp. 2754-2761, 2008.

[15] G. D. Bending and M. K. Turner, "Interaction of biochemical quality and particle size of crop residues and its effect on the microbial biomass and nitrogen dynamics following incorporation into soil," Biology and fertility of soils, vol. 23, no. 3, pp. 319-327, 1999.

[16] P. Garnier, C. Nel, C. Aita, S. Recous, F. Lafolie, and B. Mary, "Modelling carbon and nitrogen dynamics in a bare soil with and without straw incorporation," European Journal of Soil Science, vol. 54, pp. 555568,2003

[17] J. Gignoux, J. House, D. Hall, D. Masse, H. B. Nacro, and L. Abbadie, "Design and test of a generic cohort model of soil organic matter decomposition: the SOMKO model," Global Ecology \& Biogeography, vol. 10, no. 6, pp. 639-660, 2001.

[18] S. Fontaine and S. Barot, "Size and functional diversity of microbe populations control plant persistence and long-term soil carbon accumulation," Ecology Letters, vol. 8, pp. 1075-1087, 2005.

[19] N. Marilleau, C. Cambier, A. Drogoul, E. Perrier, J. Chotte, and E. Blanchart, "Multiscale mas modelling to simulate the soil environment: Application to soil ecology," Simulation Modelling Practice and Theory, vol. 16, no. 7, pp. 736-745, 2008.

[20] A. Korichi and B. Belattar, "Towards a web based simulation groupware: Experiment with BSCW,' Information Technology Journal, vol. 7, no. 2, pp. 332-337, 2008

[21] C. ElliS, S. Gibbs, and G. Rein, "Groupware some issues and experiences," COMMUNiCATiONS OF THE ACM, vol. 34, no. 1, pp. 38-58, January 1991.

[22] E. Berglund and H. Eriksson, "Distributed interactive simulation for group-distance exercise on the web," in In Proceedings of the 1998 International Conference on Web-Based Modeling Simulation. International, 1998, pp. 91-95.

[23] C. Bravo, M. A. Redondo, M. Ortega, and M. F. Verdejo, "Collaborative distributed environments for learning design tasks by means of modelling and simulation," J. Netw. Comput. Appl., vol. 29, no. 4, pp. 321-342, 2006

[24] C. Robert, "Simulation with GPSS/H," in WSC '98: Proceedings of the 30th conference on Winter simulation. Los Alamitos, CA, USA: IEEE Computer Society Press, 1998, pp. 235-240.

[25] R. Allan, X. Yang, A. Fish, M. Gonzalez, and R. Crouchley, "Using sakai for e-research: Building a multi-institution virtual research environment," 10 2007, presentation.

[26] M. North, N. Collier, and J. Vos, "Experiences creating three implementations of the repast agent modeling toolkit," ACM Transactions on Modeling and Computer Simulation, vol. 16, no. 1, pp. 1-25, 2006.

[27] U. Wilensky and I. Evanston, "Netlogo. center for connected learning and computer based modeling," Northwestern University, Tech. Rep., 1999. 